\title{
Subacute thyroiditis post-Pfizer-BioNTech mRNA vaccination for COVID-19
}

Correspondence should be addressed to F Khan

Email

dr.faroukkhan@gmail.com

\section{Summary}

There is emerging evidence of an association between COVID-19 vaccination and subacute thyroiditis. We present the case of a 42-year-old female healthcare worker who was diagnosed with subacute thyroiditis 4 days after receiving her second dose of Pfizer-BioNTech vaccine. Her clinical course followed the classical pattern for thyroiditis with spontaneous return to euthyroidism at 6 months post-presentation. The autoimmune/inflammatory syndrome induced by adjuvants has been implicated as a cause of autoimmune conditions post-vaccination and is a potential mechanism for subacute thyroiditis in our case.

\section{Learning points:}

- Subacute thyroiditis should be considered in all patients who receive any kind of vaccine for COVID-19 and subsequently develop symptoms or signs of hyperthyroidism or neck pain.

- Subacute thyroiditis is a self-limiting condition, and recognising it is important as no specific thyroid treatment (antithyroid drugs or thyroid hormone replacement) is necessary for most patients.

- The autoimmune/inflammatory syndrome induced by adjuvants may be an under-recognised cause of endocrinopathies and should particularly be considered post-vaccination.

\section{Background}

Subacute thyroiditis is an uncommon cause of thyrotoxicosis most commonly seen in women aged 20-50. It typically presents with a painful, tender thyroid gland along with classic symptoms related to the hyperthyroid state such as palpitations, tremors and weight loss. It is a self-limiting condition which typically follows a triphasic clinical course of thyrotoxicosis, hypothyroidism and return to normal thyroid function. It often follows an acute viral illness. In some patients, no treatment is required. For many patients, symptomatic relief can be achieved with non-steroidal anti-inflammatory agents while steroids are reserved for more severe cases. Beta blockers may also be used for symptomatic treatment in the thyrotoxic phase.

The autoimmune/inflammatory syndrome induced by adjuvants (ASIA) was first described by Shoenfeld and Agmon-Levin in 2011 as an entity that incorporates diverse autoimmune conditions induced by the exposure to various adjuvants (1). An adjuvant is an immunological or pharmacological substance or group of substances that can be added to a given agent to enhance efficacy and potency. Exposure to adjuvants can occur via a variety of methods due to their wide range of use in vaccines, immunisation products, mineral oils, silicone implants and many other medical products and devices. Adjuvants are generally well tolerated, but in a small number of genetically susceptible and predisposed subjects, the administration of adjuvants may trigger an autoimmune response. This may occur by a variety of mechanisms including alteration of host immunity, polyclonal activation of B cells, effects on cellular immunity, viral-induced antibodies and acceleration of molecular mimicry (2). A number of endocrine autoimmune disorders have been described as 
part of the autoimmune inflammatory syndrome induced by adjuvants, with subacute thyroiditis being one of the endocrine disorders observed (3).

It is well established that subacute thyroiditis may be associated with COVID-19 infection. Our case is only the second case of subacute thyroiditis following PfizerBioNTech mRNA vaccination for COVID-19 described in the literature. Recently, cases of subacute thyroiditis have been described post-CoronaVac, Moderna and AstraZeneca vaccines. This case highlights the need for vigilance for this condition, given the ongoing vaccine rollout worldwide and likely requirement for booster vaccines in the future.

\section{Case presentation}

A 42-year-old female healthcare worker presented to the Emergency Department with fever, palpitations and a painful left-sided neck swelling 4 days after receiving her second dose of Pfizer-BioNTech mRNA vaccine against COVID-19. She gave a history of similar symptoms 2 days after her first vaccine dose, 4 weeks prior, but on that occasion, the neck pain and swelling was right sided. She had attended her GP with these symptoms, post her first vaccine, and thyroid function tests (TFTs) showed subclinical hyperthyroidism (thyroid-stimulating hormone (TSH) $0.135 \mathrm{mIU} / \mathrm{L}$ and free thyroxine (T4) 21.5 pmol/L). Her symptoms had resolved over a couple of days with oral paracetamol and ibuprofen. The patient also had a history of asymptomatic COVID-19 infection 3 months prior to her first vaccine dose. She had not developed any symptoms in the intervening period prior to her first vaccine. She had a family history of maternal hypothyroidism.

At presentation to the Emergency Department, she was tachycardic and had a left-sided thyroid swelling which was tender on palpation. She was admitted with a clinical suspicion of thyroiditis.

\section{Investigation}

TFTs performed on admission confirmed hyperthyroidism with a suppressed TSH $<0.005 \mathrm{mIU} / \mathrm{L}$ (reference range: 0.27-4.2 mIU/L) and elevated free T4 $35.3 \mathrm{pmol} / \mathrm{L}$ (reference range: $12-22 \mathrm{pmol} / \mathrm{L}$ ). Inflammatory markers were elevated with C-reactive protein (CRP) 91 $\mathrm{mmol} / \mathrm{L}$ (reference range: $<5 \mathrm{mmol} / \mathrm{L}$ ) and erythrocyte sedimentation rate (ESR) $60 \mathrm{~mm} / \mathrm{h}$ (reference range $<20$ $\mathrm{mm} / \mathrm{h}$ ). Her anti-TPO antibodies were $10 \mathrm{IU} / \mathrm{mL}$ (reference range: $0-34 \mathrm{IU} / \mathrm{mL}$ ) and anti-TSH receptor antibodies were $<1.10 \mathrm{IU} / \mathrm{L}$ (reference range: $<1.75 \mathrm{IU} / \mathrm{L}$ ). A neck ultrasound was performed which showed a diffusely heterogeneous thyroid with multiple hypoechoic nodules demonstrating both peripheral and internal vascularity. We referred her for thyroid scintigraphy at another centre which was performed as an outpatient 4 weeks later. This was reported as being consistent with thyroiditis of the left lobe of thyroid in the right clinical and biochemical settings.

\section{Treatment}

On admission, the patient was treated with oral prednisolone $30 \mathrm{mg}$ daily in view of her significant clinical symptoms. This was continued for 2 days. She also commenced on oral propranolol LA $80 \mathrm{mg}$ daily. She reported a significant improvement in her symptoms and was discharged home on the beta blocker and oral ibuprofen $400 \mathrm{mg}$ three-times daily (TDS). A few days postdischarge she reported an increase in pain and swelling of her thyroid again and was prescribed a further short course of oral prednisolone by her GP which resulted in resolution of her symptoms.

\section{Outcome and follow-up}

Repeat TFTs 4 weeks post-discharge showed an improvement with TSH $0.093 \mathrm{mIU} / \mathrm{L}$ and free T4 15.9 $\mathrm{pmol} / \mathrm{L}$. She had also noted a significant improvement in her symptoms. TFTs were then repeated after a further 2 months which showed subclinical hypothyroidism with TSH $4.840 \mathrm{mIU} / \mathrm{L}$ and free T4 $13.4 \mathrm{pmol} / \mathrm{L}$. After a further 2 months, 5 months post her presentation, her TSH had normalised at $2.94 \mathrm{mIU} / \mathrm{L}$.

\section{Discussion}

The devastating, global COVID-19 pandemic led to one of the most rapid developments of vaccines in history. Monitoring and reporting side effects of vaccines is crucial, particularly with a rapid, worldwide rollout of a new vaccine. A number of different COVID-19 vaccines have been developed, many of which work via different mechanisms.

The COVID-19 vaccine from Pfizer-BioNTech is an mRNA-based vaccine using lipid nanoparticles to facilitate the transport of mRNA into cells. This vaccine contains a number of excipients and lipids, one of them based on polyethylene glycol (PEG). PEG may act as an adjuvant and induce an immune response in predisposed individuals. Reactions to PEG are relatively rare but have been reported, 
with PEG deemed the likely cause of anaphylaxis to the Pfizer mRNA vaccine (4).

The exact mechanism for post-vaccination subacute thyroiditis, however, remains unknown. While adjuvants contained in vaccines may play a role in producing diverse autoimmune and inflammatory responses, subacute thyroiditis has also been reported with other COVID-19 vaccines which do not contain PEG. A possible crossreactivity between thyroid cell antigens and the spike protein of the coronavirus produced by the mRNA vaccines has also been suggested (5).

A small number of cases of subacute thyroiditis following SARS-CoV-2 vaccination have been reported recently $(6,7,8,9,10,11)$. Only one previous case of subacute thyroiditis post-Pfizer-BioNTech vaccine has been described in the literature with clinical and biochemical improvement in symptoms after a short course of antiinflammatory therapy (6). A series of three cases have been described post-CoronaVac vaccine, which contains inactivated SARS-CoV-2 virus (7). Şahin Tekin et al. have also reported a case of subacute thyroiditis in a 67-year-old male patient following CoronaVac vaccine, considering the viral antigens in the COVID-19 vaccine as a possible trigger (8). Oyibo has reported a case of subacute thyroiditis following SARS-CoV-2 vaccination with AstraZeneca (9). Two more cases of subacute thyroiditis have been described after SARS-CoV-2 vaccination with Moderna Biotech and AstraZeneca (10). One other case has been described post-AstraZeneca vaccine, which contains recombinant replication-deficient chimpanzee adenovirus vector encoding the SARS CoV-2 spike glycoprotein (11).

The potential for other autoimmune thyroid diseases post-COVID-19 mRNA vaccination is highlighted by the report of two cases of new onset Graves' disease in female healthcare workers who received a Pfizer-BioNtech vaccine (12). Both developed classical clinical and biochemical findings of Graves' disease with ASIA felt to be the causative factor.

Our case adds to the emerging evidence of an association between COVID-19 vaccination and subacute thyroiditis, which has now been described with four different vaccines. As our patient had a COVID-19 infection 4 months before the presentation, the possibility of the infection itself acting as a possible trigger must be considered, but we believe the time course indicates vaccination as the more likely trigger. The cases described to date do not provide us with sufficient data to determine if there are predisposing factors, or specific risk groups (such as individuals in postpartum period), for development of subacute thyroiditis post-COVID-19 vaccines. Booster vaccination programmes are now being considered and rolled out worldwide, and we do not yet know the risk of recurrence of subacute thyroiditis in these patients. The question also arises as to whether these patients should be revaccinated with the same vaccine or a different type of vaccine. We do not believe that there is sufficient data to answer this question at the current time. It is important that further cases of subacute thyroiditis post-COVID-19 vaccines are reported to increase our knowledge and awareness, given the likely ongoing requirement for vaccination and the potential for inappropriate treatment if subacute thyroiditis is not considered.

Declaration of interest

The authors declare that there is no conflict of interest that could be perceived as prejudicing the impartiality of the research reported.

\section{Funding}

This work did not receive any specific grant from any funding agency in the public, commercial, or not-for-profit sector.

\section{Patient consent}

Written informed consent for publication of their clinical details and/or clinical images was obtained from the patient.

Author contribution statement

$\mathrm{F} \mathrm{K}$ and $\mathrm{M} \mathrm{J} \mathrm{B} \mathrm{were} \mathrm{both} \mathrm{involved} \mathrm{in} \mathrm{preparation} \mathrm{of} \mathrm{the} \mathrm{manuscript.} \mathrm{All} \mathrm{co-}$ authors have reviewed and approved the article before submission.

\section{Acknowledgements}

The authors thank the patient for granting permission to publish this information.

\section{References}

1 Shoenfeld Y \& Agmon-Levin N. 'ASIA' - autoimmune/inflammatory syndrome induced by adjuvants. Journal of Autoimmunity 2011 36 4-8. (https://doi.org/10.1016/j.jaut.2010.07.003)

2 Watad A, David P, Brown S \& Shoenfeld Y. Autoimmune/inflammatory syndrome induced by adjuvants and thyroid autoimmunity. Frontiers in Endocrinology 20167 150. (https://doi.org/10.3389/ fendo.2016.00150)

3 Bragazzi NL, Hejly A, Watad A, Adawi M, Amital H \& Shoenfeld Y. ASIA syndrome and endocrine autoimmune disorders. Best Practice and Research: Clinical Endocrinology and Metabolism 202034101412. (https://doi.org/10.1016/j.beem.2020.101412)

4 Sellaturay P, Nasser S, Islam S, Gurugama P \& Ewan PW. Polyethylene glycol (PEG) is a cause of anaphylaxis to the Pfizer/BioNTech mRNA COVID-19 vaccine. Clinical and Experimental Allergy 202151 861-863. (https://doi.org/10.1111/cea.13874) 
5 Vojdani A \& Kharrazian D. Potential antigenic cross-reactivity between SARS-CoV-2 and human tissue with a possible link to an increase in autoimmune diseases. Clinical Immunology 2020217 108480. (https:// doi.org/10.1016/j.clim.2020.108480)

6 Franquemont S \& Juan Galvez J. Subacute thyroiditis after mRNA vaccine for covid-19. Journal of the Endocrine Society 20215 A956-A957. (https://doi.org/10.1210/jendso/bvab048.1954)

7 İremli BG, Şendur SN \& Ünlütürk U. Three cases of subacute thyroiditis following SARS-CoV-2 vaccine: post-vaccination ASIA syndrome. Journal of Clinical Endocrinology and Metabolism 2021106 2600-2605. (https://doi.org/10.1210/clinem/dgab373)

8 Şahin Tekin M, Şaylısoy S \& Yorulmaz G. Subacute thyroiditis following COVID-19 vaccination in a 67-year-old male patient: a case report. Human Vaccines and Immunotherapeutics 2021 1-3. (https://doi. org/10.1080/21645515.2021.1947102)
9 Oyibo SO. Subacute thyroiditis after receiving the adenovirus-vectored vaccine for coronavirus disease (COVID-19). Cureus 202113 e16045. (https://doi.org/10.7759/cureus.16045)

10 Bornemann C, Woyk K \& Bouter C. Case report: Two cases of subacute thyroiditis following SARS-CoV-2 vaccination. Frontiers in Medicine 20218 737142. (https://doi.org/10.3389/ fmed.2021.737142)

11 Ratnayake GM, Dworakowska D \& Grossman AB. Can COVID-19 immunisation cause subacute thyroiditis? Clinical Endocrinology 2021. (https://doi.org/10.1111/cen.14555)

12 Vera-Lastra O, Ordinola Navarro A, Cruz Domiguez MP, Medina G, Sánchez Valadez TI \& Jara LJ. Two cases of Graves' disease following SARS-CoV-2 vaccination: an autoimmune/inflammatory syndrome induced by adjuvants. Thyroid 202131 1436-1439. (https://doi. org/10.1089/thy.2021.0142)

Received in final form 11 November 2021

Accepted 30 November 2021 